\title{
Best Practices in Specialty Pharmacy Management
}

\author{
Courtney J. Patterson, PharmD, BCOP
}

\begin{abstract}
BACKGROUND: Specialty pharmacy is a growing area of research, utilization, and cost. Because of the unique nature of the diseases treated by specialty pharmaceuticals, such as cancer and rheumatoid arthritis, novel management approaches are needed. Advocate Physician Partners (APP) is an entity within the Advocate Health Care Health System in the Chicago and the central Illinois area. It coordinates the care management and managed care contracting between the Advocate Health Care System and more than 4,000 physicians on the medical staffs of Advocate hospitals.

APP has experienced a per-member-per-month (PMPM) increase of $<3 \%$ in oncology intravenous medications spend in 2012. This spend refers to the intravenous medications covered under the medical benefits for APP's health maintenance organization (HMO) population. The spend has consistently been less than national projections, and we believe this is tied to the adoption of several key best practices. Prior to instituting the best practices, the yearly percentage increases for oncology spending were $5.52 \%$ (2007 to 2008), 9.39\% (2008 to 2009), and 5.29\% (2009 to 2010). After instituting best practices during the first quarter of 2011, the increases in PMPM were $3.11 \%$ (2010 to 2011) and 2.11\% (2011 to 2012), which were below previous years.
\end{abstract}

OBJECTIVE: To describe the best practices of specialty pharmacy management adopted by APP, specifically (a) establishing a content expert and governing bodies, (b) ensuring compliance with policies, and (c) providing educational resources.

METHODS: APP has several key result areas (KRAs). One KRA was compliance with appropriate utilization of intravenous oncology protocols for its HMO population. The protocols for each medication outline the appropriate indication and patient population. These protocols were developed and reviewed by the APP Pharmacy and Technology (P\&T) committee. The P\&T-approved indications reflect FDA indications and indications found in national guidelines. The APP KRA target for the utilization of protocols was $80 \%$. The compliance for completing the protocols that correspond to these medications was calculated by tabulating the number of paid claims over the number of completed protocols, resulting in a compliance percentage. APP defined noncompliance as any utilization not outlined in the protocol.

Another KRA was physician feedback. APP requires that physicians complete a certain number of continuing medical education (CME) programs provided by APP each year. Feedback from physicians, provided at the end of the CME, were tabulated and utilized for further programs. Additionally, APP strived to increase physician engagement by placing them in key roles that oversaw clinical and business aspects of the organization.

In order to meet the KRAs, investigate inappropriate utilization, and become better involved in engaging oncology specialists, APP invested in an oncology clinical pharmacist position. The primary responsibility of the oncology clinical pharmacist was to be a specialty drug resource for the organization with a focus on provider education. The oncology clinical pharmacist was deemed the content expert who developed clinical protocols, educated providers, and encouraged compliance with organizational policies and procedures as it pertained to the KRAs.

RESULTS: Since establishing the oncology clinical pharmacist position, APP has seen an increase in protocol compliance. Prior to the institution of this position, the protocol compliance was $62 \%$. In less than 1 year after hiring the oncology clinical pharmacist, the compliance percentage spiked to over $80 \%$. APP has several committees and boards that oversee the clinical and business aspects of the organization. By placing physicians in chairmanship positions of the committees, APP has seen the benefit of handling difficult patient decisions and increased compliance with policies. Lastly, extensive provider education has led to an improved physician satisfaction in the educational initiatives of APP. Greater than $80 \%$ of the physicians felt the CME content was relevant to their practices; the content would likely have a positive impact on their practices; and the clinical content was evidence based and accepted by the medical community.

CONCLUSIONS: By hiring an oncology clinical pharmacist, engaging physicians by placing them in key roles, and providing more specialist-specific education, APP has improved its KRAs and compliance percentages. APP achieved success in containing expenditures for oncology intravenous medications by implementing key best practices combined with traditional management strategies.

J Manag Care Pharm. 2013;19(1):42-48

Copyright $\odot$ 2013, Academy of Managed Care Pharmacy. All rights reserved.

\section{What is already known about this subject}

- The cost of specialty medication is increasing, and the need for management strategies is needed

- The per-member-per month medical and pharmacy benefit costs of specialty medications are increasing.

\section{What this study adds}

- This practice model provides 3 approaches employed by Advocate Physician Partners (APP) for the cost containment of oncology intravenous medications

- This practice model describes methods employed by APP to decrease inappropriate utilization of oncology intravenous medications.

$\square$ pecialty pharmacy is one of the fastest growing areas of research, utilization, and cost. The top 3 specialty therapy classes are inflammatory conditions, multiple sclerosis, and cancer. Other specialty therapy areas of high use and growth are human immunodeficiency virus (HIV), growth deficiency, anticoagulants, hepatitis $\mathrm{C}$, transplant, respiratory conditions, and pulmonary hypertension. There has been tremendous growth in the number of new therapies that have been approved. However, these new choices come at a substantial cost. It is forecasted that specialty drug spend will increase about $20 \%$ annually and will consume $40 \%$ of the drug budget by $2016 .^{1}$ 
Advocate Physicians Partners (APP) is an entity in the Advocate Health System. Advocate Health has 10 hospitals throughout the Chicago and central Illinois area. Since its inception in the mid-1990s, APP has been responsible for the care management and managed care contracting for over 4,000 physicians on the medical staff at the Advocate hospitals. APP, with approximately 130,000 health maintenance organization (HMO) lives, has contracts with several payers and is at full risk to manage certain populations. APP has also become one of the first accountable care organizations (ACO) in the country. Thus, APP is considered a hybrid of a physician health organization (PHO), managed care organization (MCO), and an ACO. Reducing the cost and ensuring appropriate utilization of specialty medications is a challenge within a large system such as Advocate Health Care. With over 2,700 specialists, greater than $50 \%$ of whom are independent physicians, variance in practice styles can create obstacles to implementing specialty programs. This article describes APP's best practices in managing specialty medications. These best practices are (a) creating an oncology clinical pharmacist position to ensure compliance with policies, (b) engaging providers through placement in advisory roles throughout the organization, and (c) providing educational resources to providers.

Methods

\section{The Oncology Clinical Pharmacist}

In 2010, APP's clinical leadership felt it important to expand the pharmacy department to assist in the execution of specialty medication programs. The organization had witnessed success in the generic medication voucher program, increasing the number of generics and vouchers used within a short time period, and wished to build upon the success of this program. ${ }^{2}$ Consequently, a full-time oncology clinical pharmacist position was created to ensure appropriate use of specialty/ oncology medications and to minimize variance in how these therapies were utilized.

This oncology clinical pharmacist position became crucial to efforts to control pharmaceutical expenditures and improve the practice of evidence-based medicine (EBM). The oncology clinical pharmacist utilized the principles of academic detailing in working with APP physicians and staff, including gastroenterology, hematology/oncology, and rheumatology specialists. The oncology clinical pharmacist was responsible for developing and maintaining specialty/oncology-related protocols; promoting and measuring compliance with those protocols; and developing and providing tools and education related to specialty/oncology medications for physicians, staff, and patients. In addition, the oncology clinical pharmacist served as a consultant to physicians, staff, and other pharmacists within APP.

As mentioned above, the oncology clinical pharmacist was responsible for developing and maintaining specialty/oncology protocols, as this is a key result area (KRA) for APP. The KRA was defined by the oncology protocol compliance percentage. The protocol compliance percentage was calculated by dividing the number of appropriately followed protocols over the number of total paid claims. The oncology protocol compliance KRA target was $80 \%$ for all physicians that prescribed medications with a protocol. The protocols served as prior authorizations (PAs), and the physicians had to detail the indication being used. The protocols were available online in APP's Electronic Referral Management Application (ERMA). To improve the oncology protocol compliance percentage, the oncology clinical pharmacist developed a communication program promoting the appropriate use of ERMA protocols specifically targeted at clinic staff who were underutilizing ERMA. The communication plan included displays in the clinic offices, speaking engagements at clinician staff meetings, and in-service classes on how to properly utilize the ERMA system.

To determine if the physicians were appropriately completing protocols in ERMA, an oncology protocol compliance report was performed quarterly. This report showed the oncology compliance percentage and reported the total number of appropriately followed protocols over the total number of paid claims for that quarter. During the quarter that the oncology clinical pharmacist was hired, the protocol compliance percentage was $62 \%$. After hiring the oncology clinical pharmacist as a content expert to educate the physicians and nurses about the importance of completing protocols as one method of ensuring appropriate utilization, there was an increase in the protocol compliance percentage to $100 \%$. Completing the protocols drives appropriate utilization because it requires physicians to choose approved indications only. In the event an unapproved indication for a drug is needed, the protocol is suspended until the case is reviewed by the PHO medical director. Thus, the protocol compliance percentage can be linked to ensuring appropriate utilization of these specialty medications. Prior to the hiring of an oncology clinical pharmacist, physicians were completing "general chemotherapy protocols" (protocols reserved for study medications or miscellaneous drugs) that were automatically approved in ERMA, wherein physicians did not have to specify an indication. By encouraging physicians to choose the appropriate medication and not the general chemotherapy protocol, appropriate utilization increased. The oncology clinical pharmacist worked with physicians and their staff to not only utilize the appropriate protocols but also to use the protocol that requires the physician to provide an indication for treatment.

The oncology clinical pharmacist has also become a clinical resource within the health system. Through collaborative work with the Pharmacy and Therapeutics (P\&T) committee, the oncology clinical pharmacist developed drug review 
summaries for all new specialty drugs. These drug summaries were distributed to all pharmacists within the organization and to physicians in the PHOs as a tool to increase access to specialty drug information. Many other clinicians within the organization look to the oncology clinical pharmacist as a resource for drug information and assistance with clinical decision making. The oncology clinical pharmacist has reviewed several difficult cases and provided crucial clinical opinions, which has greatly facilitated the APP process of approving and denying claims. Two examples of the clinical pharmacist's role in this process are highlighted by case scenarios involving the drugs bortezomib and pertuzumab.

In December 2011, the APP referral department received a PA request for bortezomib. All medication protocols completed in ERMA were reviewed by the referral department. When the indications match with the patient information already in the system, such as diagnosis, the protocol is approved, and the office is given clearance to administer the medication and then bill APP. In the event that questions arise about the indication, route, or other factors related to the appropriateness of the protocol, the referral department suspends the approval for that protocol. In this case, the referral department was hesitant to approve this medication because the route of administration was listed as subcutaneous. The initial reaction was to deny the request and instruct the physician to administer the medication by the traditional intravenous route. However, the referral department consulted the oncology clinical pharmacist for information about the bortezomib route of administration. The pharmacist researched the question and found an abstract that was presented at the American Society of Hematology in 2010 and a study that was published in 2011 by Moreau et al. investigating intravenous versus subcutaneous routes for bortezomib. ${ }^{3,4}$ This clinical information and review of details about the patient case led to the approval of the request and subsequent approval of the claim. In January 2012, the U.S. Food and Drug Administration (FDA) approved the subcutaneous route of administration, which provided some assurance of the oncology clinical pharmacist's value within a managed care organization to assist in the decision matrix of approving and denying of protocols.

In June 2012, an APP PHO medical director was presented with a patient case for pertuzumab. Pertuzumab was approved in May 2012 for the treatment of patients with human epidermal growth factor receptor 2 (HER2)-positive metastatic breast cancer who have not received prior anti-HER2 therapy or chemotherapy for metastatic disease. Pertuzumab must be given with trastuzumab and docetaxel. ${ }^{5}$ The patient was a 52-year-old female with metastatic breast cancer with metastasis to the liver and bone. She had received previous therapies in the nonmetastatic setting, including heavy radiation. Two months prior to the request for the medication, she had started on trastuzumab and docetaxel. At the time of the claim for pertuzumab, she had received 2 doses of trastuzumab. Both the medical director and the referral department consulted the oncology clinical pharmacist for assistance with approving or denying the request. The pharmacist reviewed the exclusion criteria in the study by Baselga et al. (2012) with the medical director and the prescribing physician. ${ }^{6}$ In this study, the patients had to have not had anti-HER2 therapy for 12 months. The patient had only received 2 months of trastuzumab therapy and had recently become metastatic. Patients in the clinical trial who had received trastuzumab (in the nonmetastatic setting) still benefited from pertuzumab. The pharmacist recommended that the pertuzumab be approved for the patient. The pharmacist used the manufacturer's medical information department to obtain information about this off-label use, which was incorporated into the recommendation. The prescribing physician, medical director, and oncology clinical pharmacist collaborated to form a clinical recommendation to approve the drug request. With the approval of the medication, the patient was able to access a medication that has been shown to extend progression free survival. Although there are a number of different ways that these two drug information inquiries could have been addressed, the common denominator was that the availability of an oncology clinical pharmacist to the PHO physicians, PHO medical directors, and referral department had improved the quality of the care provided.

\section{Collaboration of Governing Bodies and Decision Making}

APP has several governing bodies that have both clinical and business oversight of the organization. The Utilization Management (UM) committee oversees best practices and appropriate utilization for all aspects of health care. A subset of the UM committee is the P\&T committee, which develops programs, policies, and procedures related to pharmaceuticals, with a more recent focus on specialty and oncology medications. In addition, the P\&T committee is responsible for reviewing PA protocols, new medications, and drug utilization reviews. The P\&T committee's mission is to promote the appropriate use of pharmaceuticals based on the principles of EBM with membership composed of the following physician specialties: cardiology, family medicine, internal medicine, hematology/oncology, pediatrics, and rheumatology. The committee also invites guest physicians when they are reviewing an item pertaining to a specialty different from the committee members.

Streamlining new medication reviews, clinical policy development, and clinical decision making through one governing body has been essential to APP's success in ensuring that EBM is practiced throughout the system. An example of the impact of this best practice is illustrated by the decision APP made on bevacizumab utilization for breast cancer. 


\section{FIGURE 1 Advocate Physician Partners ERMA Oncology Protocol Compliance}

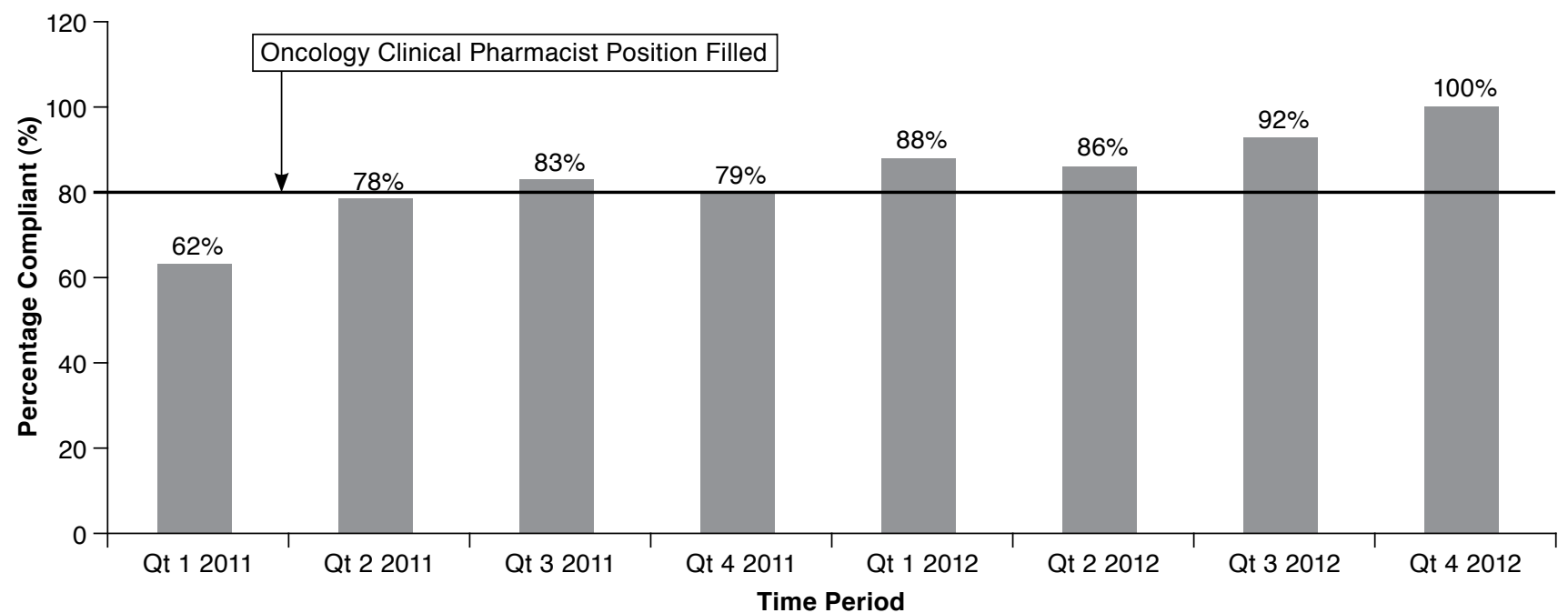

$\mathrm{ERMA}=$ electronic referral management application

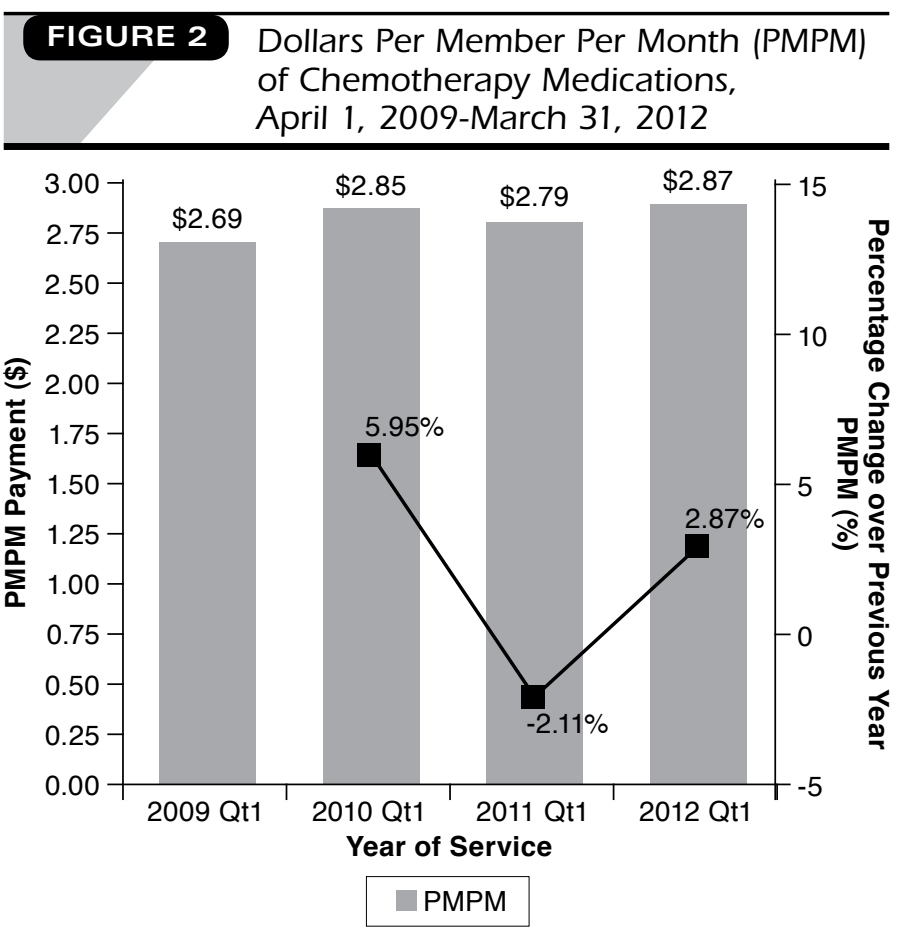

In January 2011, the APP P\&T committee made the decision to remove the breast cancer indication from the bevacizumab protocol. The decision was made just after the Center for Drug Evaluation and Research (CDER) proposal to withdraw the indication for metastatic breast cancer from the bevacizumab drug label. The P\&T committee's decision, made before that of the FDA, was based on a review and analysis of two clinical trials. Robert et al. (2011) conducted a phase III, randomized, placebo-controlled trial comparing bevacizumab in combination with several standard chemotherapy regimens versus those regimens alone for first-line treatment of patients with HER2negative metastatic breast cancer. Although progression-free survival (PFS) was seen, an improvement in overall survival (OS) was not demonstrated. ${ }^{7}$ Miles et al. (2010) conducted a randomized, phase III study comparing bevacizumab in combination with docetaxel versus docetaxel alone in women with HER2 negative, previously untreated, metastatic breast cancer. ${ }^{8}$ Both of these trials were designed to confirm bevacizumab's impact on overall survival. However, the difference in PFS with the addition of bevacizumab, the lack of OS improvement, and the toxicities of the drug were considered by the P\&T committee. The FDA made a decision in November 2011 to retract the breast cancer indication. However, between January and November 2011, the utilization of bevacizumab in breast cancer had already dropped to zero for APP. At the time, the National Comprehensive Cancer Network (NCCN) still listed bevacizumab as a treatment option, and physicians could continue to use the medication, as it was supported by the NCCN guidelines. ${ }^{9}$ However, because of physician education, the large influence of the P\&T committee, and pharmacist involvement within the APP PHO, there continues to be no utilization of bevacizumab in breast cancer treatment. 


\begin{tabular}{ll}
\hline TABLE 1) & $\begin{array}{l}\text { Value Added Services Provided } \\
\text { by Oncology Clinical Pharmacist } \\
\text { (July 2011-July 2012) }\end{array}$ \\
\hline Services Provided & Quantity \\
\hline Claims denial and approval consultations & 7 \\
\hline Drug information questions answered & 23 \\
\hline Drug summaries sent to providers & 6 \\
\hline Educational presentations & 11 \\
\hline Newsletter publications & 12 \\
\hline In-office physician visits & 31 \\
\hline P\&T presentations provided & \\
\hline PET = pharmacy and therapeutics. & \\
\hline
\end{tabular}

\section{Provider Education}

Provider education is a valuable way to improve the quality of care delivered and to achieve organizational goals. Physician engagement is one of the KRAs for Advocate Health Care, and one way APP engages its physicians is by providing them with educational opportunities. In the fall of 2011, the APP Pharmacy Services department developed a physician continuing medical education (CME) program for all APP physicians. This CME program provided an update on changes within the pharmacy landscape for 2011. The CME material covered new generic medications, anticipated generics for 2011, landmark clinical trials, vaccination updates, FDA warnings, and new drug indications and formulations divided by specialty.

In addition to the CME program, the pharmacy team developed drug summary sheets that were used to provide additional physician education. The drug information summaries were developed by the oncology clinical pharmacist and approved by the P\&T committee. After P\&T approval, the drug summary sheets were included with the provider newsletter and highlighted drug details, clinical trial information, administration, and dose adjustment details. Because APP feels that more informed providers will lead to improved prescribing habits and well-informed patients, it has provided a variety of education opportunities for its providers and encouraged provider education in various formats.

\section{Results}

One of the best practices of APP was creating the oncology clinical pharmacist position. This pharmacist was responsible for developing and maintaining specialty/oncology-related protocols. The efforts to educate and collaborate with physicians and their staff have resulted in APP achieving its KRAs. The organizational target for compliance with the oncology protocols has consistently been above $80 \%$ since the implementation of staff education in this area, thus, demonstrating the value of a subject matter expert liaison to specialty clinics (Figure 1).

Additionally, the emphasis on getting physicians to appro-

\section{TABLE 2 Impact of Provider Education: Survey Results, January-December 2012 $(N=690)$}

\begin{tabular}{l|c|c}
\hline Satisfaction Rates - Physician Feedback & $\begin{array}{c}\text { Strongly } \\
\text { Agree (\%) }\end{array}$ & $\begin{array}{c}\text { Agree } \\
\text { (\%) }\end{array}$ \\
\hline $\begin{array}{l}\text { Teaching methods were appropriate for } \\
\text { the subject matter }\end{array}$ & 51.19 & 45.83 \\
\hline $\begin{array}{l}\text { Clinical content was evidence-based and accepted } \\
\text { by the medical community }\end{array}$ & 49.11 & 48.11 \\
\hline Content was relevant to my practice & 41.95 & 44.93 \\
\hline Content is likely to have an impact on my practice & 41.24 & 46.00 \\
\hline Stated objectives were met & 51.30 & 46.31 \\
\hline Faculty communicated effectively & 47.90 & 49.50 \\
\hline Overall, this was an excellent activity & 44.89 & 47.70 \\
\hline
\end{tabular}

priately complete protocols has led to a decrease in inappropriate utilization and minimized increases in annual drug spend. Implementation of staff education may have contributed to reduction in expected increase in expenditures for intravenous chemotherapy medications (Figure 2). The annual growth in per-member-per-month (PMPM) was 5.95\% in 2010 and 2.87\% in 2012 (first quarter data compared annually). Of note, in 2011, the annual growth in PMPM was $-2.11 \%$. Although the exact cause is unknown, this was the year in which robust implementation of staff education and appropriate protocol utilization procedures began.

In addition to the case scenarios discussed, the number of services the oncology clinical pharmacist provided to physicians and patients within APP is summarized in Table 1. These services included visiting physician offices for in-services, providing education to increase compliance with organizational policies, and contributing to the APP monthly provider newsletter, which highlights new drug developments. The value of services provided by the oncology clinical pharmacist was one contributing factor in reaching the organizational KRA goals and demonstrating improvement in the protocol review process.

APP has seen positive results from providing physicians with a variety of educational opportunities in various formats. The CME program received overwhelmingly positive feedback with $80 \%$ of 680 participants responding that the information was valuable and could possibly impact their practices (Table 2). In addition to the CME program, Table 3 highlights the other types of educational initiatives produced from June 2011 to July 2012.

A limitation of our dataset is that the claims data represents a small portion of the APP commercial products and highlights only the HMO utilization. However, the numbers, while small, consistently show improved protocol compliance, minimal offlabel utilization, and improved physician engagement in educational opportunities. As shown in the case of bevacizumab, 


\begin{tabular}{l|l}
\hline \multicolumn{1}{c}{ TABLE 3 } & $\begin{array}{l}\text { Types of Provider Education Tools } \\
\text { (June 2011-July 2012) }\end{array}$ \\
\hline Type of Education & \multicolumn{1}{c}{ Details } \\
\hline CME & $\begin{array}{l}\text { Drug Summary 2011 published in online CME por- } \\
\text { tal for APP physicians. }\end{array}$ \\
\hline Drug Summary 2012 in development and slated for \\
a February 2013 release.
\end{tabular}

in an organization as large as APP, no off-label use of this drug demonstrates the impact of physician education and communication. The collaboration between the oncology pharmacist, the P\&T committee, other physicians, and decision makers within the health system has led to positive results.

\section{Conclusions}

Many organizations, including APP, manage specialty medications with policies, procedures, and rules. This study provided insight into one organization's management approach to the use of specialty pharmaceuticals. The best practices of APP center on having an oncology clinical pharmacist with a strong clinical background who acts as a content expert, integrating clinical services with those of specialist providers and serving as a clinical drug resource within the organization Other steps that have helped drive appropriate drug utilization included the establishment of a governing body with physician presence and provision of value-added provider services, such as CME programs. The combined modalities have led to a $100 \%$ oncology protocol compliance percentage in quarter 4 of 2012, which resulted in APP meeting its KRA target of $80 \%$. Combining these methods with traditional management strategies may lead to the successful management of specialty medications. We believe that our best practices model contributed to reduction in expected increase in expenditures for chemotherapy. The data also showed a reduction in the percentage increase in oncology drug expenditures every year from 2009 to 2012. These results are limited in scope and time, as they do not include patient outcomes data and capture only our HMO population. However, we are encouraged by these positive trends and plan to continue to employ our best practices model to ensure appropriate use of specialty medications.

\section{Author}

COURTNEY J. PATTERSON, PharmD, BCOP, is Oncology Clinical Pharmacist, Advocate Physician Partners, Oak Brook, Illinois.

AUTHOR CORRESPONDENCE: Courtney J. Patterson,

PharmD, BCOP, 2025 Windsor Dr., Oak Brook, IL 60523.

Tel.: 630-990-5570; Fax: 630-990-5499;

E-mail:courtney.patterson@advocatehealth.com.

\section{DISCLOSURES}

The author reports no financial conflicts of interest related to the subjects discussed in this article and has received no financial incentive for authorship.

\section{REFERENCES}

1. Express Scripts. 2011 drug trend report. Express Scripts Research \& New Solutions Lab. April 2012. Available at: http://www.express-scripts.com/ research/research/dtr/archive/2012/dtrFinal.pdf. Accessed January 26, 2013.

2. Bhargava V, Greg M, Shields M. Addition of medication vouchers to a pharmacist academic detailing program: effects on the generic dispensing ratio in a physician-hospital organization. J Manag Care Pharm. 2010;16(6):384-92.

3. Moreau P, Pylypenko H, Grosicki S, et al. A phase 3 prospective randomized international study (MMY-3021) comparing subcutaneous and intravenous administration of bortezomib in patients with relapsed multiple myeloma. Blood. 2010;116(21):Abstract 312

4. Moreau P, Pylypenko H, Grosicki S, et al. Subcutaneous versus intravenous administration of bortezomib in patients with relapsed multiple myeloma: a randomised, phase 3, non-inferiority study. Lancet Oncol. 2011;12(5):431-40. 
5. Perjeta (pertuzumab). [package insert]. Genentech. Revised June 2012. Available at: http://www.gene.com/download/pdf/perjeta_prescribing.pdf. Accessed January 29, 2013.

6. Baselga J, Cortés J, Sung-Bae Kim, et al. Pertuzumab plus trastuzumab plus docetaxel for metastatic breast cancer. N Engl J Med. 2012;366(2):109-19.

7. Robert NJ, Diéras V, Glaspy J, et al. RIBBON-1: randomized, double-blind, placebo-controlled, phase III trial of chemotherapy with or without bevacizumab for first-line treatment of human epidermal growth factor receptor 2-negative, locally recurrent or metastatic breast cancer. J Clin Oncol. 2011;29(10):1252-60.
8. Miles D, Chan A, Dirix L, et al. Phase III study of bevacizumab plus docetaxel compared with placebo plus docetaxel for the first-line treatment of human epidermal growth factor recepter-2 negative metastatic breast cancer. J Clin Oncol. 2010;28(20):3239-47.

9. National Comprehensive Cancer Network. NCCN guidelines for the treatment of cancer by site: breast cancer. (v3 2012). Available at: http://www. nccn.org/professionals/physician_gls/f_guidelines.asp\#breast. Accessed January 31, 2013. 